\title{
Embodied Intersubjectivity and the Vitality of Cultural Meaning: Narratives of Communicative Musicality in Learning and Teaching
}

\author{
Stephen Malloch ${ }^{1}$, Jonathan Delafield-Butt ${ }^{2}$, Colwyn Trevarthen ${ }^{3}$ \\ 1. Stephen Malloch, Honorary Fellow, Westmead Psychotherapy Program, University of \\ Sydney, Sydney, Australia; Adjunct Fellow, MARCS Institute for Brain, Behaviour and \\ Development, Western Sydney University, Sydney, Australia. \\ stephen.malloch@heartmind.com.au
}

2. Professor of Child Development \& Director of the Laboratory for Innovation in Autism, University of Strathclyde, Glasgow, Scotland, United Kingdom. jonathan.delafieldbutt@strath.ac.uk

3. Professor (Emeritus) of Child Psychology and Psychobiology, University of Edinburgh, Edinburgh, Scotland, United Kingdome. c.trevarthen@ed.ac.uk

\author{
PREPRINT \\ $9^{\text {TH }}$ JULY 2019
}

\begin{abstract}
Human learning is inspired with the purposes and feelings of individuals who seek conscious, in-the-moment cooperation. It is social and co-created through mutual attunement of the movements of body and mind. In school, the interested learner needs to be encouraged by a skilled teacher sensitive to the rhythms of the child's friendly, open vitality. They co-create shared projects in play, with movement and language, developing meaning and learning in sympathetic collaboration. From infancy, projects of imagination are expressed by the body and voice with the creative forms of 'communicative musicality' - gestural narratives created in rhythms of movement, felt, seen and heard. They anticipate being responded to with love and care. Learning within these embodied narratives incorporates affective, energetic, and intentional components to produce schemas of engagement that structure knowledge, and become meaningful habits held in memory. The rituals of culture and technical skills develop from the psycho-motor structure of human nature, with its vital impulses of thought-in-action that express an integrated, imaginative, and sociable Self.
\end{abstract}




\section{Introduction}

We are animals motivated to explore with energetic curiosity, to move towards people and objects with hopeful expectation, and to move away with fear when the possibility of harm is anticipated. We interact with trusted companions, enjoying play, testing boundaries in the safety of supportive and caring relationships (Panksepp and Trevarthen, 2009; Trevarthen and Delafield-Butt, 2015). Our consciousness is the ongoing creation of Self-awareness in a coherent system of rhythmic brain activity, generating knowledge as we engage with the world, and applying our learnings from the ways we manipulated it for curiosity and pleasure (Trevarthen and Delafield-Butt, 2017). An act of thinking represents movement, of body parts or of objects and people, represented as perceptions and ideas in the mind of a single agent (Llinás, 2001).

Our knowledge of the world, coming from our motivated movement as we explore it, has its basis in our evolutionary past (Llinás, 2001). Intrinsic to movement is the exploration of the future - animals need to predict what might come next so they can move with safety and creativity through the world. This embodied intentionality appears in human life at the end of the first trimester of gestation (see Chapter X on 'Infant Intentionality' in this encyclopedia). From the start, plans for action grow with the 'serial ordering' of simple, separate actions, from which develops our logical reasoning and the grammar of language. 'Patterns of action' of music, dance, ritual and language (Llinás, 2001) are revealed by close observation of the 'intrinsic motive pulse' infants share in intimate communication with loving caregivers. Our innate skill for moving, remembering and planning projects in sympathy with others arises from coherence of brain activity in time. It has been called 'communicative musicality' because this rhythmic, collaborative, future exploring activity is clearly expressed in the human creation of music and dance motivated by a desire to communicate and collaborate with and understand others (Malloch and Trevarthen, 2009).

\section{The communicative musicality of infants' early experience}

A baby is born with an awareness of another person's body as having feelings in movement like their own proprioceptive and visceral ones, which regulate rhythms of well-being. From this innate 'musicality' shared with companions comes mastery of human culture and language (Vygotsky, 1967). A child learns with pride in social participation. Skill and knowledge in cultural practice is brought to life with the enthusiastic support of more experienced companions.

Communicative musicality has three dimensions (Malloch and Trevarthen, 2009). First, its PULSE is a regular succession through time of discrete movements, to create rhythms of music, of dance, expressive vocalizations and collaborative movements that create meaningful human interactions. These movements are made using a felt sense of acting that enables a 'future-creating' predictive process by which a person may anticipate or create what happens next, and when. Second, these movements are formed with a QUALITY composed of the contours of expressive vocal and body gesture, shaping one's felt sense of the passage of time expressed in movement. These contours can consist of psychoacoustic attributes of a vocalization - timbre, pitch, volume - or attributes of acceleration, speed, direction and intensity of the moving body perceived, felt, viewed, or heard. Quality communicates the affective meaning within an embodied, expressed 
intention. This quality later becomes richly associated with events and meanings that express a conceptual intelligence. Finally, these expressive movements compose NARRATIVES of individual experience and of companionship built from sequences of gestures co-created with others, each made with the pulses and qualities that altogether bring aesthetic pleasure.

\section{Narratives - strategies of purpose - form the structure of experience and learning}

Co-created narratives of body movements, made within the intrinsic vitality of the psychomotor timings of the body and nervous system, are synchronized within and between subjects by what music professor Nigel Osborne describes as the 'chronobiology' of intelligent movement (Osborne, 2009). The elements of active embodied consciousness are combined in rhythmic projects that become adventurous locomotions of the body, tasks of object manipulation, or narratives of communication by gesture or vocalization. Osborne outlines three levels of energy or initiative in the 'present moment' of conscious movement and communication, summarized as follows:

Quick and Important (a frequency of around five movement pulses per second): Attentions and signals to get things done in the world as it is, and to share it. Reaching to grasp or use something. For example, a nod, glance, eyebrow raise, wave, burst of laughter, or grimace; stepping to walk or to run. A musical pulse or beat represented by andante to presto. A stressed syllable in poetry or a full vowel.

Interesting, Skilled, Informative (a frequency of around one movement per second): Relaxed. A single conscious action within short-term memory; a reach, pointing for showing, with a short word or call. Working at a comfortable pace, sawing for example. A slow walk. A 'foot' in poetry. Musical pulse represented by largo to andante

Slow, Careful (a frequency of around one movement every two seconds): The selfaware purposeful present, with relaxed breathing. An oculomotor scan path or manipulative sequence. A long turn in conversation or long word; a phrase in song or poetry. A slow gesture or utterance in a story-making manner; sedate and kind.

A narrative structure common to all the time-based arts of music, dance, drama, and poetry emerges in social engagements (Figure 1) (Malloch and Trevarthen, 2009; Trevarthen and Delafield-Butt, 2013). A four-part story-line of introduction, development, climax, and resolution enables a single self-created or co-created unit of meaning-making that can be held in memory to be shared, enabling anticipation of future similar events in private moments of vitality, in intimacy with one particular person, or with a whole group of companions (Stern, 2010).

From the beginning of life, narratives are evident within the interactions between mothers, fathers, caregivers and their babies (Delafield-Butt and Trevarthen, 2015). And this universal form of motivation for meaning making remains through human development. 


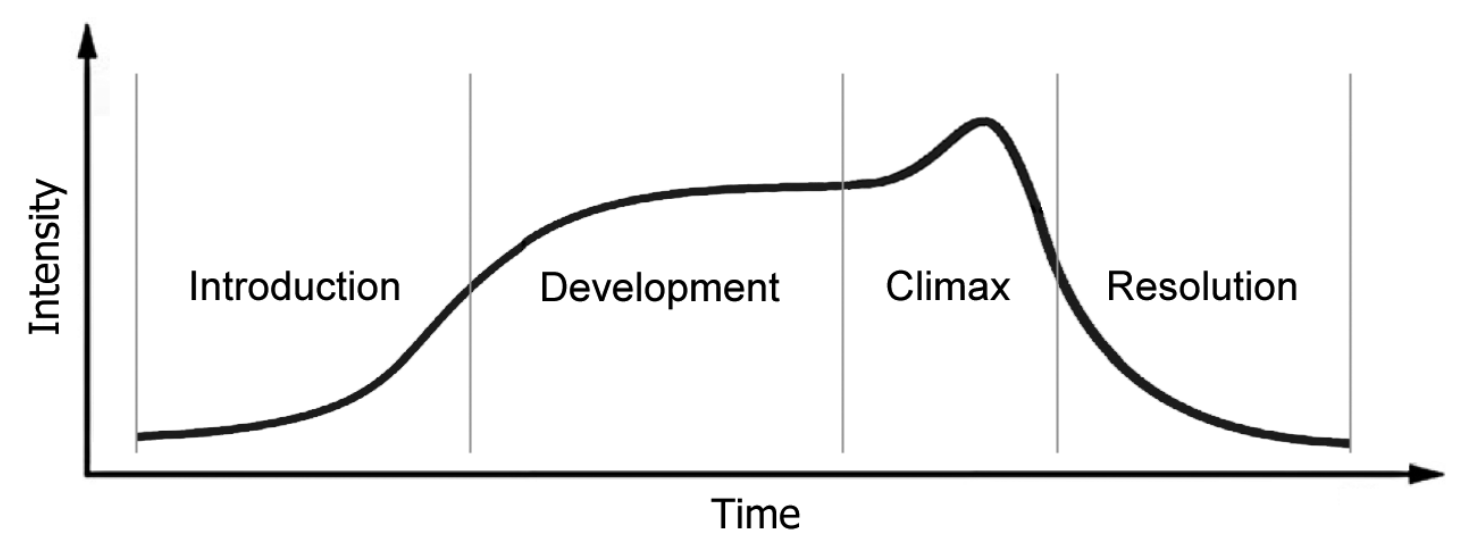

Figure 1: Intensity contour of impulses to move. This is a narrative over four phases: (i) 'interest' in the narrative begins at a low-intensity in the introduction, which 'invites' participation in purposefulness; (ii) the coordination of the actions and interests of real and imagined agents intensifies over the development, as the 'plan' or 'project' develops; (iii) a peak of excitation with achievement of a goal in single or mutual intention is reached at the climax, after which (iv) the intensity reduces as the purpose of the participant(s) is to share a resolution, and those who were closely engaged, separate. Reproduced with permission from Trevarthen and Delafield-Butt (2013).

\section{Infant leadership}

Infants stimulate an affectionate caregiver, male or female, to extended poetic or musical speech, which often moves into wordless song, or imitative, rhythmic and repetitive nonsense sounds. This distinctive style of adult speech is called 'motherese,' 'parentese,' 'infant directed speech' (IDS), or 'infant inclusive speech' (IIS), and is attended to and responded to with much pleasure by infants. It varies with the age and state of the infant, and reflects the infant's motives and emotions.

Caregivers' speech to infants has unconscious or intuitive forms that are common across cultures. The earliest shared signing in infancy becomes dialogic 'musical babbling' or 'proto-conversation' from around 2 months of age. The baby starts to learn the cultural gestures and preferences that become the tools for creating cultural meanings.

\section{The importance of transactions of feeling in co-created embodied narratives}

Affect attunement, 'the performance of behaviours that express the quality of feeling of a shared affect state, but without imitating the exact behavioural expression of the inner state' (Stern et al. 1985, p.142), is an unfolding quality of feeling expressed in a form of behavior that has a 'vitality contour'. This largely unconscious 'recasting' of events is necessary to 'shift the focus of attention to what is behind the behavior, to the quality of feeling that is being shared' (Stern et al. 1985, p.142).

This is much more than mimicking. Mimicking implies we are attentive to a person's behavior (an important factor in building a relationship) but it tells the other person very little, if anything, about the inner state of the person who mimics. However, to abstract a 
component from those movements, and relay it back to the initiator in a changed yet recognizable form, says we are creating with something that has been given to us by the other. I am externalizing some of my own inner life while in relationship. The relationship is now one of companionship (from the Latin meaning 'to break bread with', and defined here as the wish to be with an other for a mutually beneficial 'inner' purpose, apart from reasons of immediate survival, procreation or material gain). Companionship involves exchanging affect through sharing impulses of motivation.

Infants have no language to learn what other humans know through practical experience. But the vitality of their spontaneous communicative musicality or prosody, highly coordinated and adapted to be shared through narratives with sympathetic and playful companions, enables meaningful communication, which builds memories - learning extended in space and time - 'autonoetic' thoughtful consciousness.

Intrinsic to the sociability of this intelligence of movement is sensitivity for the exploration of the future. Karl Lashley, reflecting on the evolution of animal movement, proposed that the ability to predict what might come next, and to plan the 'serial ordering' of separate actions, may be understood as the foundation for our logical reasoning as an individual, as it is for the grammar or syntax and prosody of language. A restless futureseeking intelligence, with our urge to share it, inspires us to express our personalities as 'story-telling creatures', who want to share, and evaluate, others' stories (Bruner, 1996).

\section{The role of early education}

Our creativity comes from the way our body hopes to move, with rhythms and feelings of grace and biological 'knowing', as identified by the phenomenological philosopher Merleau-Ponty. The predicting, embodied self of a human being experiences time, force, space, movement, and intention/directionality in being. Together, these form the Gestalt of 'vitality' by which we sense in ourselves and in others that bodily movement, with thought and affective feeling, is 'well-done' (Trevarthen and Malloch, 2017).

As children grow, informal learning of this 'well-done' leads to the creation of their own children's culture inspired by the vitality of their innate communicative musicality. Then, if early education does its job well, with the infant as collaborative creator, artist and thinker, our rich inner narrative of life expressed in our social group creates a lifeaffirming, inclusive culture of rituals that celebrate the aesthetic grace and moral graciousness of a creative life. The education of an infant is one of shared attention that values each movement and meaning. This shared attention values the particular, the individual nuance of movement and meaning. It does not try to standardise in a prescribed way - rather it celebrates the novel, and leads the infant, and later the child, to express herself in ways that come from the particular motivations of her own intrinsic wish to move in the world, and create new shared meanings.

This balance has been presented as a consideration of two Latin roots for the English word 'education.' One, educare, means to train or to mould. Its motivation is the initiation of a person into cultural conventions, without which a person is left unable to live effectively within a particular culture, using its tools to communicate. The other, educere, means to lead out, or draw out. Without this more responsive nurturing, the person is left 
unable to engage with situations and solve problems not yet imagined. Their ability for creativity is compromised. These very different concepts of what education means are often experienced in schooling as being in tension, with educare often winning out, leaving the child with dry knowledge rather than living abilities supported by their own innate skills.

School is a social environment where a child's own skills and abilities can be nurtured, and a place where training is at hand to provide entrance into the ways of a particular culture (Trevarthen, Delafield-Butt, and Dunlop, 2018). The success of teaching to help form a healthy, resilient life depends on recognition of how the inborn 'zest for learning' is present across the lifespan, enabled by shared, co-created stories of meaning that form a child's mind, and that of our culture.

\section{References}

Bruner, J. S. (1996). The Culture of Education. Cambridge, MA.: Harvard University Press.

Delafield-Butt, J. T., and Trevarthen, C. (2015). The Ontogenesis of Narrative: From moving to meaning. Frontiers in Psychology, 6. doi:10.3389/fpsyg.2015.01157

Llinás, R. (2001). I of the Vortex: From Neurons to Self. Cambridge MA: MIT Press.

Malloch, S., \& Trevarthen, C. (2009). Musicality: Communicating the vitality and interests of life. In S. Malloch and C. Trevarthen (Eds.), Communicative Musicality: Exploring the basis of human companionship (pp. 1-12). Oxford: Oxford University Press.

Osborne, N. (2009). Towards a chronobiology of musical rhythm. In Malloch, S. and Trevarthen, C. (eds.) Communicative Musicality: Exploring the Basis of Human Companionship, 545-564. Oxford: Oxford University Press.

Panksepp, J. and Trevarthen, C. (2009). The neuroscience of emotion in music. In Malloch, S. and Trevarthen, C. (eds), Communicative Musicality: Exploring the Basis of Human Companionship,_105-146. Oxford: Oxford University Press.

Stern, D. N. (2010). Forms of Vitality: Exploring Dynamic Experience in Psychology, the Arts, Psychotherapy and Development. Oxford: Oxford University Press.

Stern, D. N., Hofer, L., Haft, W. and Dore, J. (1985). Affect attunement: The sharing of feeling states between mother and infant by means of inter-modal fluency. In Field, T. M. and Fox, N. A. (eds) Social Perception in Infants. Norwood, N.J.: Ablex, 249-268.

Trevarthen, C., \& Delafield-Butt, J. T. (2017). Intersubjectivity in the Imagination and Feelings of the Infant: Implications for Education in the Early Years. In E. J. White \& C. Dalli (Eds.), Under-three Year Olds in Policy and Practice (pp. 17-39). New York: Springer. 
Trevarthen, C., \& Delafield-Butt, J. T. (2015). The Infant's Creative Vitality, In Projects of Self-Discovery and Shared Meaning: How They Anticipate School, and Make It Fruitful. In S. Robson and S. F. Quinn (Eds.), International Handbook of Young Children's Thinking and Understanding (pp. 3-18). Abingdon, Oxfordshire \& New York: Routledge.

Trevarthen, C., \& Delafield-Butt, J. T. (2013). Biology of Shared Meaning and Language Development: Regulating the Life of Narratives. In M. Legerstee, D. Haley, \& M. Bornstein (Eds.), The Infant Mind: Origins of the Social Brain (pp. 167-199). New York: Guildford Press.

Trevarthen, C. \& Malloch, S. (2017). Grace in moving and joy in sharing: the intrinsic beauty of communicative musicality from birth. In, S. Bunn, Ed. Anthropology and Beauty: From Aesthetics to Creativity. London: Taylor and Francis/Routledge, Chapter 8, pp. 115-130.

Trevarthen, C., Delafield-Butt, J., \& Dunlop, A.-W. (Eds.). (2018). The Child's Curriculum: Working with the natural values of young children. Oxford: Oxford University Press.

Vygotsky, L. S. (1967) Play and its role in the mental development of the child. Soviet Psychology, 5(3), 6-18. (Republished in, Bruner, J. S., Jolly, A and Sylva, K (eds) (1985). Play -- Its Role in Development and Evolution. Harmondsworth: Penguin.) 